\title{
Sepsis decreases the activity of acetylcholinesterase by reducing its expression at the neuromuscular junction
}

\author{
JIN WU*, TIAN JIN**, HONG WANG and SHI-TONG LI \\ Department of Anesthesiology, Shanghai General Hospital, Shanghai Jiao Tong \\ University School of Medicine, Shanghai 200080, P.R. China
}

Received November 8, 2016; Accepted June 28, 2017

DOI: $10.3892 / \mathrm{mmr} .2017 .7265$

\begin{abstract}
Our previous study demonstrated that sepsis may decrease the activity of acetylcholinesterase (AChE) at the neuromuscular junction (NMJ) of the diaphragm at $24 \mathrm{~h}$, and thus improve the antagonistic action of neostigmine on rocuronium. The present study aimed to determine the effects of sepsis on AChE activity over 2 weeks, which is a more clinically relevant time period. Furthermore, the present study aimed to elucidate the association between AChE activity and its expression at the NMJ during sepsis. Male adult Sprague-Dawley rats were randomly divided into the sham or sepsis groups. Sepsis was induced by cecal ligation and puncture. On days 1, 3, 7 and 14 after surgery, AChE activity at the NMJ of the diaphragm was detected using a modified Karnovsky and Roots method. Furthermore, AChE expression levels at the NMJ, and in the whole muscle fibers of the diaphragm, were detected by immunohistofluorescence staining and western blot analysis, respectively. AChE activity was significantly decreased in the sepsis group, with its lowest level detected on day 7; however, its activity had partially recovered on day $14(\mathrm{P}<0.01)$. AChE activity was positively correlated $(r=0.975, P=0.025)$ with its expression at the $\mathrm{NMJ}$, which showed a similar trend over 2 weeks of sepsis. The protein expression levels of $\mathrm{AChE}$ in the whole muscle fibers of the diaphragm were significantly decreased on days 1, 3 and 7 in the sepsis group $(\mathrm{P}<0.01)$, with the lowest level observed
\end{abstract}

Correspondence to: Professor Shi-Tong Li, Department of Anesthesiology, Shanghai General Hospital, Shanghai Jiao Tong University School of Medicine, 100 Hai Ning Road, Shanghai 200080, P.R. China

E-mail: lishitongs@yahoo.com

*Contributed equally

Abbreviations: AChE, acetylcholinesterase; AChR, acetylcholine receptor; CLP, cecal ligation and puncture; Erk, extracellular signal-regulated kinases; NMJ, neuromuscular junction; PBS, phosphate-buffered saline

Key words: acetylcholinesterase, neuromuscular junction, diaphragm, sepsis on day 3. In conclusion, sepsis decreased AChE activity by reducing its expression at the NMJ over 14 days; the reduced expression of AChE at the NMJ might be as a result of its reduced muscular production.

\section{Introduction}

In general anesthesia, non-depolarizing muscle relaxants are frequently used to facilitate endotracheal intubation and to make surgical procedures easier to perform (1). These muscle relaxants bind to nicotinic cholinergic receptors at the neuromuscular junction (NMJ) of skeletal muscle, however, they are unable to induce muscle depolarization, thus they competitively antagonize the neurotransmitter acetylcholine (ACh), resulting in muscle relaxation (1). Among the different types of muscle relaxants, rocuronium is favored by anesthesiologists in clinical practice as it has a rapid onset, an intermediate duration of action and a low number of side effects (2). Due to incomplete metabolism and excretion of muscle relaxants, a residual neuromuscular blockade occurs frequently during the postoperative period, which can result in muscle weakness, aspiration and even critical respiratory events $(3,4)$.

Acetylcholinesterase (AChE) is expressed throughout the $\mathrm{NMJ}$ and can rapidly hydrolyze ACh to prevent its accumulation, thereby returning the skeletal muscle to its resting state prior to the next motor nerve impulse (5). Anticholinesterase drugs, including neostigmine, are commonly administered at the end of anesthesia to reverse the residual neuromuscular block (1). By inhibiting the activity of AChE at the NMJ, these drugs decrease the hydrolysis of acetylcholine, thus increasing its concentration to overcome the effects of muscle relaxants (5). Our previous study demonstrated that acute sepsis at $24 \mathrm{~h}$ was able to strengthen the antagonistic action of neostigmine on rocuronium by inhibiting the activity of $\mathrm{AChE}$ at the NMJ in a rat cecal ligation and puncture (CLP) model (6). These results indicate that, in clinical practice, when used to reverse residual neuromuscular block, the dosage of neostigmine can be appropriately reduced in patients with sepsis, and consequently, the incidence of its side effects will decrease. These side effects include severe bradycardia, bronchospasm, copious secretions and paradoxical weakness (5). CLP-induced sepsis can be divided into the early phase, which occurs during the first 5 days, and the chronic phase, which occurs after day 5 (7). The present study aimed to assess the 
alterations in AChE activity at the NMJ over 2 weeks of sepsis, since this time period is more relevant to clinical sepsis $(7,8)$. Furthermore, the present study aimed to determine whether the decreased activity of AChE was caused by its reduced expression at the NMJ. The results will provide a preliminary theoretical basis for the rational clinical use of anticholinesterase drugs in patients with chronic sepsis.

\section{Materials and methods}

Animals and experimental design. The present study was approved by the Animal Care and Use Committee of the Shanghai General Hospital affiliated with Shanghai Jiao Tong University (Shanghai, China). Experiments were performed on male adult Sprague-Dawley rats (age, 7 weeks; weight, 220-260 g) and were purchased from the Shanghai SLAC Laboratory Animal Co., Ltd (Shanghai, China). All rats were pathogen-free and were allowed to acclimate for $\geq 1$ week prior to experimentation. The animals were given ad libitum access to food and water, and were housed at an ambient temperature $\left(23-25^{\circ} \mathrm{C}\right)$ and relative humidity $(50-70 \%)$ under a 12 -h light/dark cycle.

A total of 24 rats were randomly assigned to the sham group, which consisted of the following four subgroups ( $\mathrm{n}=6 /$ subgroup): $1 ; 3 ; 7$; and day 14 . In addition, a total of 44 rats were assigned to the sepsis group, which consisted of the following four subgroups: Day $1, n=8$; day $3, n=10$; day $7, n=12$; and day $14, n=14$. A greater number of rats were assigned to the sepsis group due to the expected increase in the rate of mortality induced by the CLP model and the extended period of observation. In the sepsis group, sepsis was surgically induced using the CLP method (9). Briefly, after the rats were anesthetized with pentobarbital $(50 \mathrm{mg} / \mathrm{kg}$, intraperitoneal injection), a midline abdominal incision was made to expose the cecum. Three-quarters length of the cecum was ligated tightly and punctured with an 18-gauge needle from the mesenteric toward the antimesenteric direction. A small amount of feces was squeezed out of the cecum through the two penetration holes. The cecum was then replaced, and the abdomen was closed in two layers. In the sham group, the rats were anesthetized in the same manner and underwent the same operation without CLP. At the end of the surgery, all rats were resuscitated with a subcutaneous administration of $10 \mathrm{ml}$ pre-warmed $\left(37^{\circ} \mathrm{C}\right)$ normal saline into the back. The rats had ad libitum access to water and food after surgery and received the antibiotic imipenem $(25 \mathrm{mg} / \mathrm{kg}$; EKEAR Biotechnology Co., Ltd, Shanghai, China) intraperitoneally every $12 \mathrm{~h}$ for $72 \mathrm{~h}$. Due to its wide antimicrobial spectrum, imipenem is able to elevate the survival of septic animals (10).

On days 1, 3, 7 or 14 after surgery, all of the rats that had survived were sacrificed by intraperitoneal injection of pentobarbital $(100 \mathrm{mg} / \mathrm{kg})$. Subsequently, the dorsal costal region of the right hemidiaphragm was collected and stored in liquid nitrogen for western blot analysis of AChE in the muscle fibers, whereas the remaining parts of the diaphragm were immediately used for the measurement of AChE activity and its expression at the NMJ.

Histological detection of AChE activity. AChE activity at the $\mathrm{NMJ}$ in the ventral costal region of the left hemidiaphragm

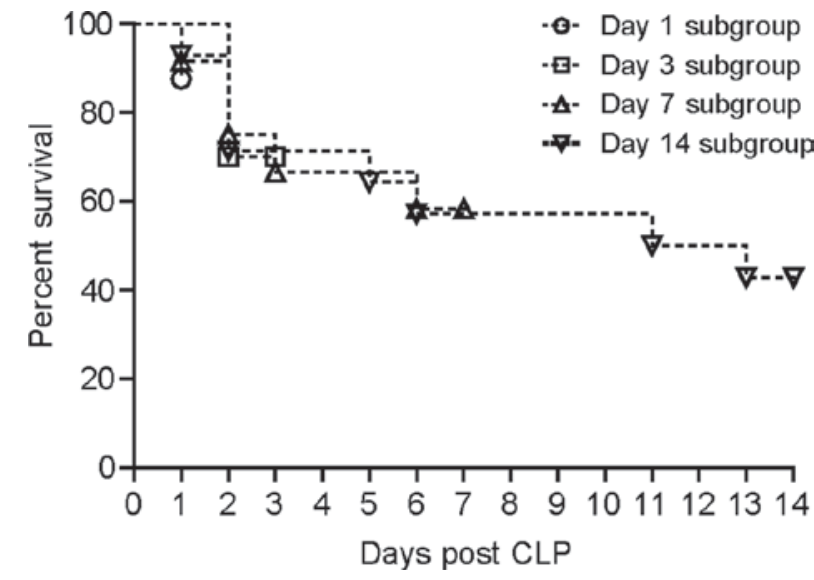

Figure 1. Survival rates following CLP in the sepsis group. At the start of the analysis, $\mathrm{n}=8,10,12$ and 14 for the day 1, 3, 7 and 14 subgroups, respectively. Of the rats that succumbed, most died within $48 \mathrm{~h}$. CLP, cecal ligation and puncture.

was detected according to a modified Karnovsky and Roots method (11). The detailed procedure was described in our previous study (6). Brown staining indicated AChE activity, which was recorded as the sum of the individual measurements of AChE activity observed at each NMJ.

Immunohistofluorescence staining of AChE. The ventral costal region of the right hemidiaphragm containing motor endplates was dissected and sectioned longitudinally to $15 \mu \mathrm{m}$ using a cryostat (Shandon Cryotome FE; Thermo Fisher Scientific, Inc., Waltham, MA, USA) at a temperature of $-20^{\circ} \mathrm{C}$. Muscle sections were then fixed in acetone for $1 \mathrm{~min}$ at room temperature. After being washed in phosphate-buffered saline (PBS), blocking was performed in PBS containing 10\% normal donkey serum (Jackson ImmunoResearch Laboratories, Inc., West Grove, PA, USA) for 30 min at room temperature. The preparations were then stained with an anti-AChE antibody (1:100; cat. no. PA5-21371; Thermo Fisher Scientific, Inc.) in the blocking solution for $20 \mathrm{~h}$ at $4^{\circ} \mathrm{C}$. After three washes with PBS (5 min/wash), the preparations were incubated with Alexa Fluor 594-conjugated donkey anti-rabbit secondary antibody (1:500; cat. no. 711-585-152; Jackson ImmunoResearch Laboratories, Inc.) and Alexa Fluor 488-conjugated $\alpha$-bungarotoxin (1:1,000; cat. no. B13422; Thermo Fisher Scientific, Inc.) for $1 \mathrm{~h}$ at room temperature. After three washes with PBS (5 min/wash), the sections were mounted with antifade mounting medium (Beyotime Institute of Biotechnology, Shanghai, China). Confocal laser scanning microscopy (TCS SP8, Leica Microsystems GmbH, Wetzlar, Germany) was used to examine the slides with excitation/emission wavelengths at 499/520 and 590/617 nm for Alexa Fluor 488 and 594 fluorophores, respectively. Images of five randomly selected visual fields containing motor endplates under 200x magnification were obtained in each muscle section. The average optical density of AChE at the NMJ was calculated using Image-Pro Plus software (version 6.0; Media Cybernetics, Inc., Rockville, MD, USA).

Western blot analysis of AChE protein expression. The dorsal costal region of the right hemidiaphragm was 
A

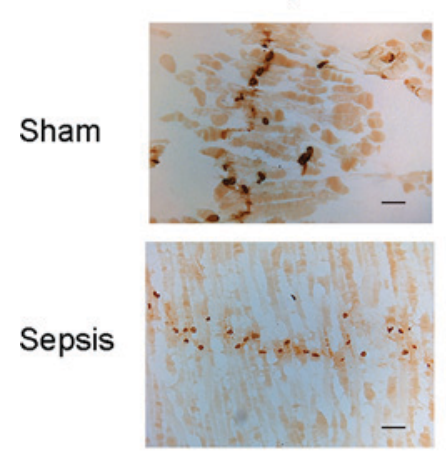

Day 3
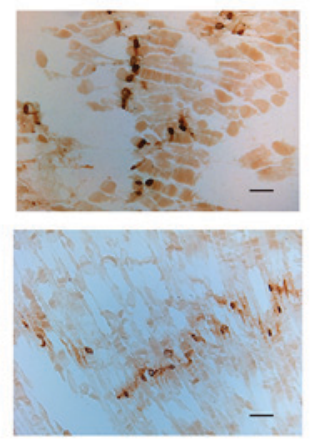

Day 7

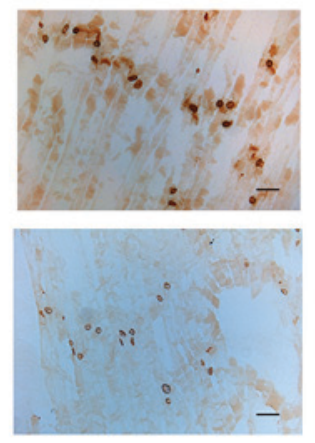

Day 14
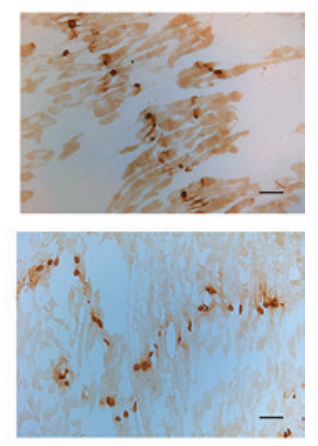

B

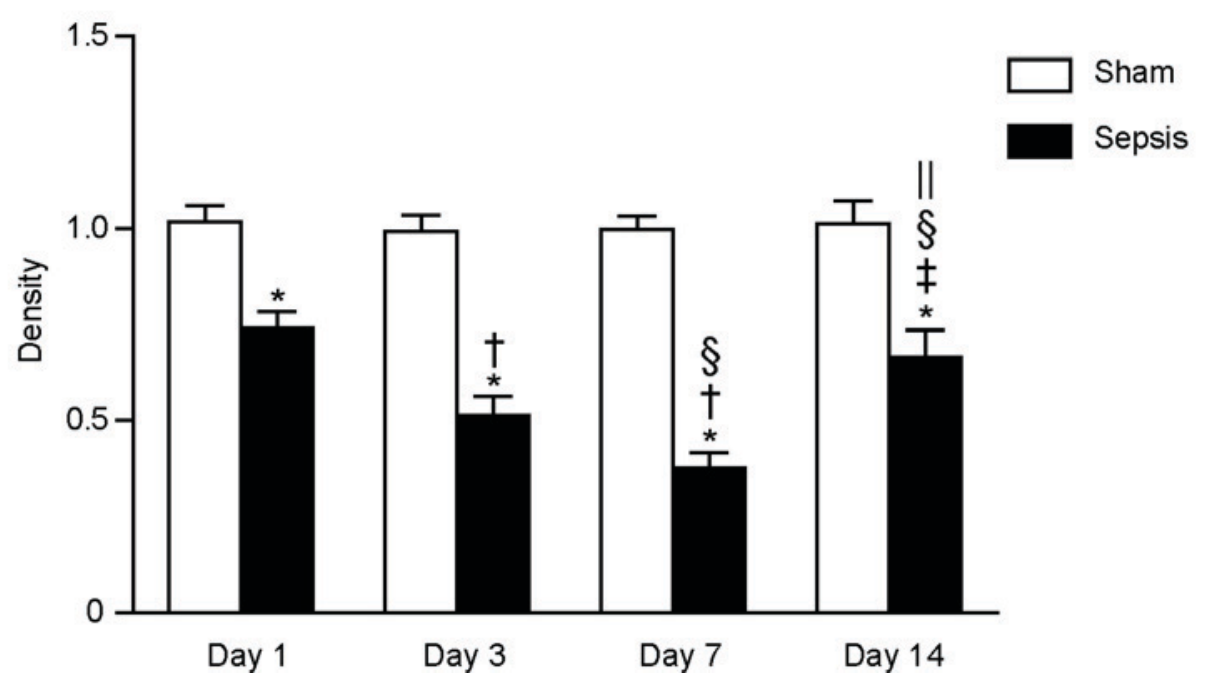

Figure 2. (A) AChE activity, as determined using a modified Karnovsky and Roots method, at the NMJ of the rat diaphragm. Scale bar, $100 \mu \mathrm{m}$. (B) Average optical density of AChE activity at the NMJ. Data are presented as the mean \pm standard deviation $(n=6)$. ${ }^{*} \mathrm{P}<0.01$ vs. the sham group; ${ }^{+} \mathrm{P}<0.05$ and ${ }^{\mathrm{T}} \mathrm{P}<0.01 \mathrm{vs}$. the day 1 sepsis subgroup; ${ }^{\circledR} \mathrm{P}<0.01$ vs. the day 3 sepsis subgroup; "P<0.01 vs. the day 7 sepsis subgroup. AChE, acetylcholinesterase; NMJ, neuromuscular junction.

homogenized in radioimmunoprecipitation lysis buffer (Beyotime Institute of Biotechnology) containing $1 \mathrm{mM}$ phenylmethanesulfonyl fluoride (Amresco, LLC, Solon, OH, USA). The protein concentration was determined using a bicinchoninic acid protein assay kit (Beyotime Institute of Biotechnology). Subsequently, $30 \mu \mathrm{g}$ protein was separated by $10 \%$ SDS-PAGE, and the proteins were transferred to a nitrocellulose membrane (EMD Millipore, Billerica, MA, USA). For immunoblotting, the membranes were blocked with $5 \%$ nonfat milk for $1 \mathrm{~h}$ at room temperature; thereafter, they were incubated overnight at $4^{\circ} \mathrm{C}$ with primary antibodies against AChE (1:500; cat. no. PA5-21371; Thermo Fisher Scientific, Inc.) and $\beta$-actin (1:1,000; cat. no. A20120A0702; Bio TNT, Shanghai, China). After washing three times in Tris-buffered saline with $0.1 \%$ Tween-20, the blots were incubated for $1 \mathrm{~h}$ with peroxidase-conjugated goat anti-rabbit secondary antibodies (1:10,000; cat. no. 111-035-003; Jackson ImmunoResearch Laboratories, Inc.) and were visualized using an electrochemiluminescence detection kit (EMD Millipore). Densitometry was performed using ImageJ version 1.48 software (National Institutes of Health, Bethesda, MD, USA). The relative protein expression levels were determined by normalization to $\beta$-actin protein expression as an internal reference, and are expressed as relative density units.
Statistical analysis. Data are presented as the mean \pm standard deviation, unless otherwise specified. Initially, the data were tested for normality and equality of variance. Subsequently, one-way analysis of variance was conducted, followed by a Bonferroni post hoc test to compare the differences at the same time point. For comparison of different time points within the same group, one-way analysis of variance was conducted, followed by a least significant difference test. The correlation between AChE activity, its expression at the NMJ and its content in whole muscle fibers in the sepsis group was determined using a Pearson correlation analysis. All statistical analyses were performed using SPSS version 17.0 statistical software (SPSS, Inc., Chicago, IL, USA). P<0.05 was considered to indicate a statistically significant difference.

\section{Results}

Survival rates postoperation. All rats in the sham group survived the preset observational periods, whereas the survival rates in the day $1,3,7$ and 14 sepsis subgroups were 87.5, 70, 58.3 and $42.9 \%$, respectively (Fig. 1). The septic rats began to develop fever, chills, tachypnea, piloerection, subconjunctival hemorrhage and diarrhea $\sim 8 \mathrm{~h}$ after the laparotomy. They became progressively more lethargic, and their movement decreased. These symptoms were most obvious on day 2 . 
A

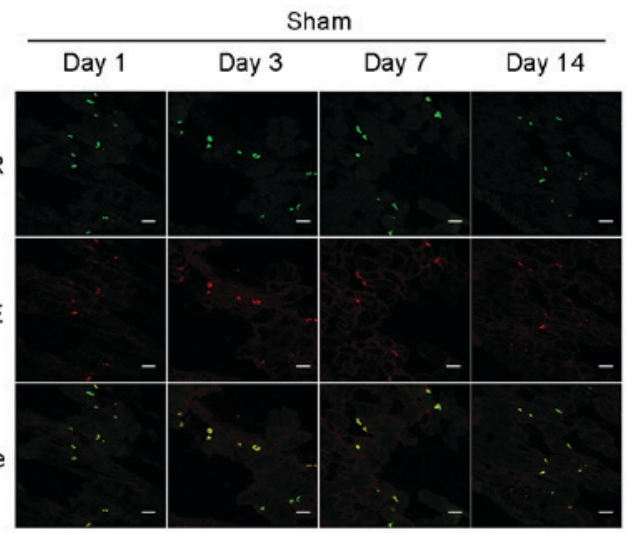

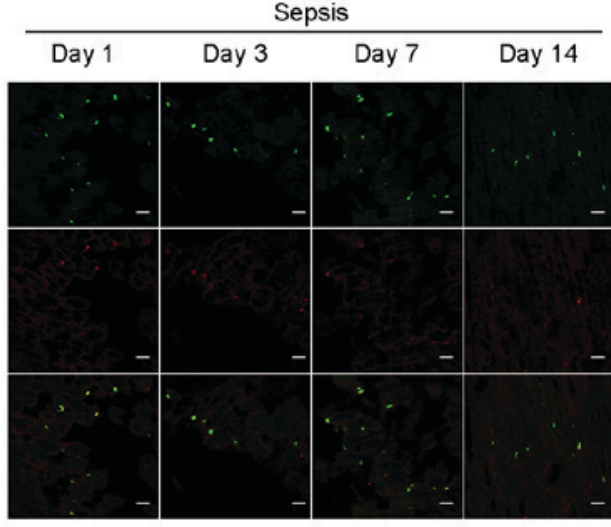

B

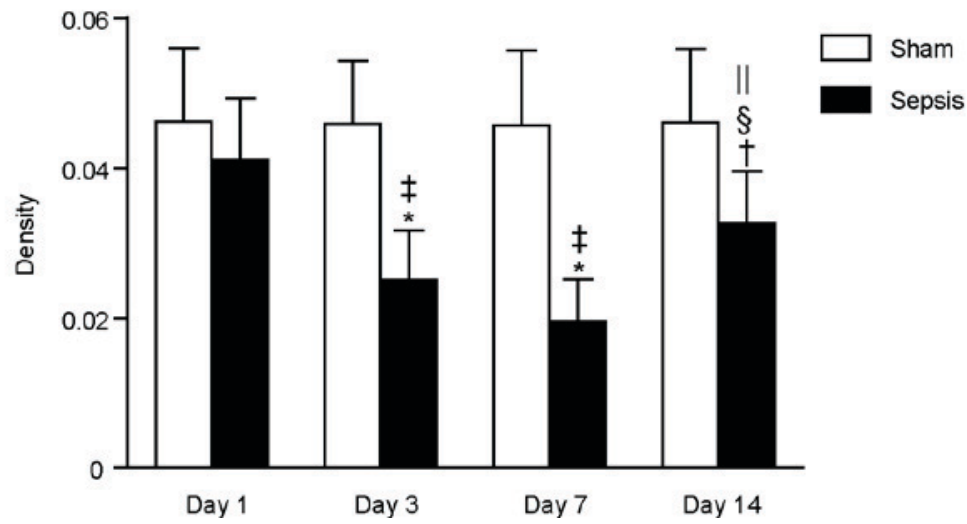

Figure 3. (A) Immunohistofluorescence staining of AChE at the NMJ of the rat diaphragm. Scale bar, $50 \mu \mathrm{m}$. Muscle sections were double-stained with Alexa Fluor 488-conjugated $\alpha$-bungarotoxin (green) to locate the NMJ and anti-AChE antibody/Alexa Fluor 594-conjugated anti-rabbit secondary antibody (red) to visualize the AChE at the NMJ. (B) Average optical density of AChE expression at the NMJ. Data are presented as the mean \pm standard deviation (n=6). ${ }^{\dagger} \mathrm{P}<0.05$ and ${ }^{*} \mathrm{P}<0.01$ vs. the sham group; ${ }^{\circledR} \mathrm{P}<0.05$ and ${ }^{\ddagger} \mathrm{P}<0.01$ vs. the day 1 sepsis subgroup; ${ }^{\prime} \mathrm{P}<0.01$ vs. the day 7 sepsis subgroup. AChE, acetylcholinesterase; $\mathrm{AChR}$, acetylcholine receptor; NMJ: neuromuscular junction.

All rats that survived to day 7 or 14 had an intra-abdominal abscess, which was walled off.

Histological staining of AChE activity. As presented in Fig. 2A, brown insoluble staining was localized to the diaphragm muscle fibers. In the sepsis group, AChE activity was significantly decreased on day 1 compared with the sham group; the lowest AChE activity was detected on day 7, whereas activity had partially recovered by day $14(\mathrm{P}<0.01$; Fig. $2 \mathrm{~B})$.

Immunohistofluorescence staining of AChE. As presented in Fig. 3A, AChE staining (red) was co-localized with acetylcholine receptor staining (green) at the NMJ. After a slight decline on day $1(\mathrm{P}>0.05)$, the expression levels of AChE were significantly decreased on day 3 compared with the sham group; the lowest AChE expression was detected on day 7 , whereas expression had partially recovered by day $14(\mathrm{P}<0.05$; Fig. 3B).

Protein expression levels of AChE in the diaphragm. The relative protein expression levels of AChE, normalized to $\beta$-actin, were significantly decreased on days 1,3 and 7 in the sepsis group compared with in the sham group $(\mathrm{P}<0.01$; Fig. 4$)$. The lowest expression was detected on day 3 . On day 14 , protein expression in the sepsis group remained lower than in the sham group; however, the difference was not significant $(\mathrm{P}>0.05)$.
Correlation between AChE activity and its expression at the NMJ during sepsis. A statistically significant positive correlation was observed between AChE activity and its expression at the $\mathrm{NMJ}$ in the sepsis group $(\mathrm{r}=0.975, \mathrm{P}=0.025$; data not shown). However, the expression of $\mathrm{AChE}$ at the NMJ was not positively correlated with the protein expression of AChE in the whole diaphragm in the sepsis group $(\mathrm{r}=0.627, \mathrm{P}=0.373$; data not shown).

\section{Discussion}

The results of the present study demonstrated that sepsis decreased AChE activity at the NMJ of the diaphragm in a rat model of CLP over 14 days. This decreased activity was observed as early as 1 day after the CLP operation, was most obvious on day 7 and partially recovered on day 14. In addition, the expression levels of $\mathrm{AChE}$ at the NMJ exhibited a similar trend over 2 weeks, and AChE activity was revealed to be positively correlated with its expression at the NMJ.

The present study observed a positive correlation between AChE activity and its expression at the NMJ during sepsis. Since the level of AChE activity detected in the present study was the sum of the individual AChE activities detected at each NMJ, the decreased activity of AChE at the NMJ during sepsis was due to reduced levels of this protein. Notably, on day 1 post-CLP, AChE activity at the NMJ was significantly 
A

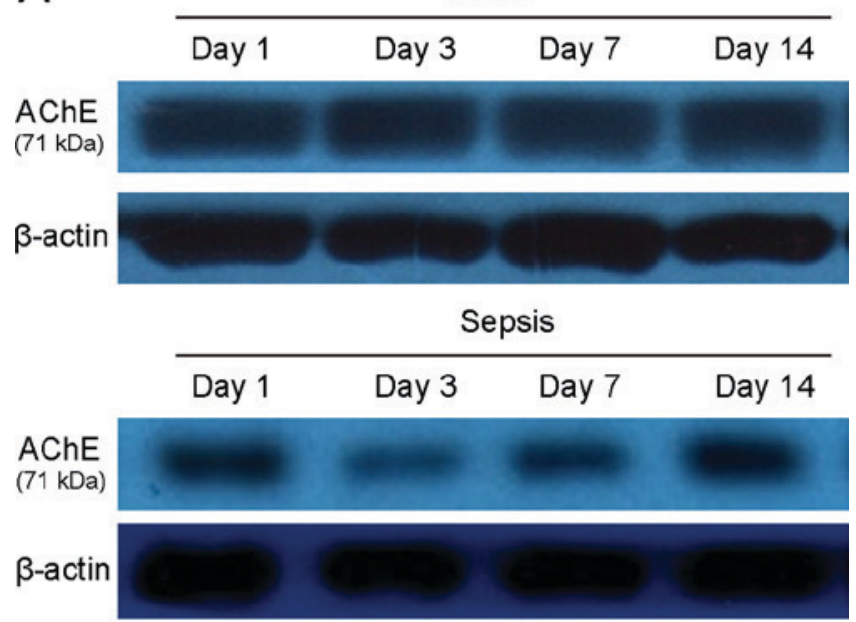

B

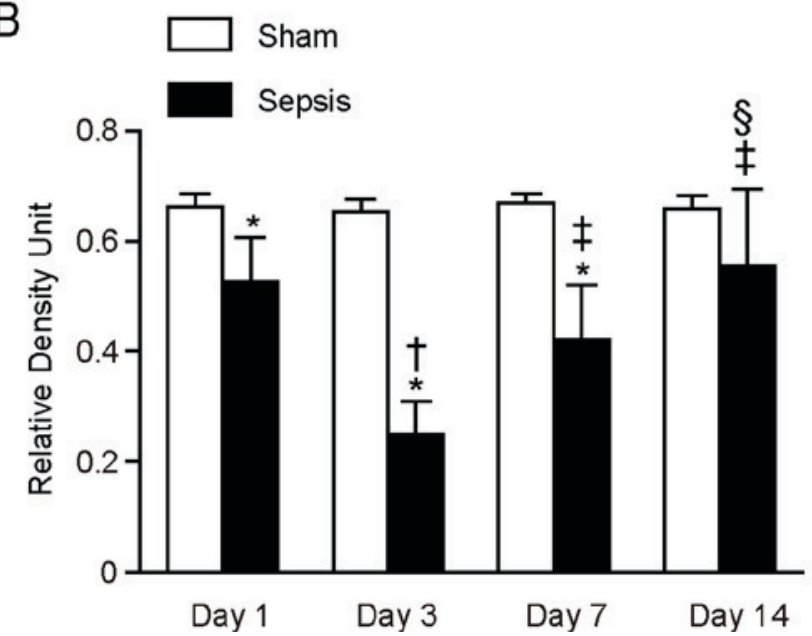

Figure 4. (A) Western blot analysis of AChE protein expression in the diaphragm. Two representative images are presented. (B) Relative expression levels of AChE normalized to $\beta$-actin. Data are presented as the mean \pm standard deviation $(\mathrm{n}=6)$. ${ }^{\mathrm{P}}<0.01$ vs. the sham group; ${ }^{\mathrm{P}} \mathrm{P}<0.01$ vs. the day 1 sepsis subgroup; ${ }^{\dagger} \mathrm{P}<0.01$ vs. the day 3 sepsis subgroup; ${ }^{\circledR} \mathrm{P}<0.05$ vs. the day 7 sepsis subgroup. AChE, acetylcholinesterase

decreased, whereas its expression was only slightly reduced. One possible explanation for this finding is that the level of AChE activity at the NMJ during the acute period of sepsis may be partially inhibited by factors such as severe acidosis $(6,12)$, hypochlorous acid $(13,14)$ or other unknown factors.

It may be hypothesized that the majority of $\mathrm{AChE}$ at the $\mathrm{NMJ}$ originates from muscle fibers (6). In the present study, it was demonstrated that alterations in the expression of $\mathrm{AChE}$ at the NMJ lagged behind the alterations in its overall content in the whole diaphragm, and the expression levels between the NMJ and the whole diaphragm were not positively correlated. This delayed phenomenon may be due to the comparatively long half-life of AChE at the NMJ. In cultured chick embryo muscles, $\sim 2 / 3$ of total AChE was located within cells. The intracellular AChE constituted a pool with rapid turnover, with a synthesis rate of $\sim 20 \%$ of the total AChE per hour; the remaining $1 / 3$ of $\mathrm{AChE}$ was located on the plasma membrane, which had a half-life of $\sim 50 \mathrm{~h}(15,16)$. Using fluorescent fasciculin 2 to specifically and selectively label AChE at the NMJ of living mice, Krejci et al (17) reported that the half-life of AChE was initially 3 days, and $\sim 1 / 3$ of AChE at the NMJ was very stable.

Our previous study demonstrated that the mRNA expression levels of AChE were lower in the diaphragm of septic rats (6). In the present study, it was further confirmed that the protein expression of AChE was reduced in septic rats. Therefore, in the present study, the reduced protein expression of AChE in the diaphragm may be due to its reduced protein synthesis. A potential mechanism is described as follows: At the NMJ, ATP is released from the presynaptic motor neuron and activates $\mathrm{P} 2 \mathrm{Y}$ receptors on the postsynaptic muscle. This process phosphorylates extracellular signal-regulated kinases (Erk) by triggering a mitogen-activated protein kinase signaling cascade. Phosphorylated Erk can then activate ELK1, ETS transcription factor, which increases the transcriptional activity of the $\mathrm{AChE}$ gene by binding to the AChE promoter $(18,19)$. However, sepsis-induced mitochondrial dysfunction may impair the generation of ATP in motor neurons (20). Furthermore, phosphorylation of Erk has previously been reported to be significantly reduced in the skeletal muscle of rats with an intra-abdominal septic abscess (21). In addition, protein synthesis during sepsis may be impaired (22).

In conclusion, the present study demonstrated that sepsis decreases the activity of AChE by reducing its expression at the NMJ. In our previous study, the antagonistic action of neostigmine on rocuronium was strengthened when AChE activity was inhibited during acute sepsis (6). Therefore, further research is required in order to elucidate the effects of varying degrees of decreased AChE activity on the efficiency of neostigmine during chronic sepsis.

\section{Acknowledgements}

The present study was supported by a grant from the National Natural Science Foundation of China (grant no. 81171845).

\section{References}

1. Bronsert MR, Henderson WG, Monk TG, Richman JS, Nguyen JD, Sum-Ping JT, Mangione MP, Higley B and Hammermeister KE: Intermediate-acting nondepolarizing neuromuscular blocking agents and risk of postoperative 30-day morbidity and mortality and long-term survival. Anesth Analg 124: 1476-1483, 2017.

2. Huh H, Park SJ, Lim HH, Jung KY, Baek SK, Yoon SZ, Lee HW, Lim HJ and Cho JE: Optimal anesthetic regimen for ambulatory laser microlaryngeal surgery. Laryngoscope 127: 1135-1139, 2017.

3. Brull SJ and Murphy GS: Residual neuromuscular block: Lessons unlearned. Part II: Methods to reduce the risk of residual weakness. Anesth Analg 111: 129-140, 2010.

4. Schreiber JU: Management of neuromuscular blockade in ambulatory patients. Curr Opin Anaesthesiol 27: 583-588, 2014.

5. Caldwell JE: Clinical limitations of acetylcholinesterase antagonists. J Crit Care 24: 21-28, 2009.

6. Wu J, Jin T, Wang H and Li ST: Sepsis strengthens antagonistic actions of neostigmine on rocuronium in a rat model of cecal ligation and puncture. Chin Med J (Engl) 129: 1477-1482, 2016.

7. Xiao H, Siddiqui $J$ and Remick DG: Mechanisms of mortality in early and late sepsis. Infect Immun 74: 5227-5235, 2006.

8. Benjamim CF, Hogaboam CM and Kunkel SL: The chronic consequences of severe sepsis. J Leukoc Biol 75: 408-412, 2004

9. Rittirsch D, Huber-Lang MS, Flierl MA and Ward PA: Immunodesign of experimental sepsis by cecal ligation and puncture. Nat Protoc 4: 31-36, 2009. 
10. Newcomb D, Bolgos G, Green L and Remick DG: Antibiotic treatment influences outcome in murine sepsis: Mediators of increased morbidity. Shock 10: 110-117, 1998.

11. Karnovsky MJ and Roots L: A 'Direct-coloring' thiocholine method for cholinesterases. J Histochem Cytochem 12: 219-221, 1964.

12. Wessler I, Michel-Schmidt $\mathrm{R}$ and Kirkpatrick CJ: $\mathrm{pH}$-dependent hydrolysis of acetylcholine: Consequences for non-neuronal acetylcholine. Int Immunopharmacol 29: 27-30, 2015.

13. Gaut JP, Yeh GC, Tran HD, Byun J, Henderson JP, Richter GM, Brennan ML, Lusis AJ, Belaaouaj A, Hotchkiss RS and Heinecke JW: Neutrophils employ the myeloperoxidase system to generate antimicrobial brominating and chlorinating oxidants during sepsis. Proc Natl Acad Sci USA 98: 11961-11966, 2001.

14. den Hartog GJ, Vegt E, van der Vijgh WJ, Haenen GR and Bast A: Hypochlorous acid is a potent inhibitor of acetylcholinesterase. Toxicol Appl Pharmacol 181: 228-232, 2002.

15. Rotundo RL and Fambrough DM: Synthesis, transport and fate of acetylcholinesterase in cultured chick embryos muscle cells. Cell 22: 583-594, 1980.

16. Rotundo RL: Expression and localization of acetylcholinesterase at the neuromuscular junction. J Neurocytol 32: 743-266, 2003.
17. Krejci E, Martinez-Pena y Valenzuela I, Ameziane R and Akaaboune M: Acetylcholinesterase dynamics at the neuromuscular junction of live animals. J Biol Chem 281: 10347-10354, 2006.

18. Xu ML, Bi CW, Cheng LK, Mak S, Yao P, Luk WK, Lau KK, Cheng AW and Tsim KW: Reduced expression of P2Y2 receptor and acetylcholinesterase at neuromuscular junction of P2Y1 receptor knock-out mice. J Mol Neurosci 57: 446-451, 2015.

19. Choi RC, Chu GK, Siow NL, Yung AW, Yung LY, Lee PS, Lo CC, Simon J, Dong TT, Barnard EA and Tsim KW: Activation of UTP-sensitive P2Y2 receptor induces the expression of cholinergic genes in cultured cortical neurons: A signaling cascade triggered by $\mathrm{Ca}^{2+}$ mobilization and extracellular regulated kinase phosphorylation. Mol Pharmacol 84: 50-61, 2013.

20. Singer M: The role of mitochondrial dysfunction in sepsis-induced multi-organ failure. Virulence 5: 66-72, 2014.

21. Vary TC, Deiter G and Lang CH: Diminished ERK 1/2 and p38 MAPK phosphorylation in skeletal muscle during sepsis. Shock 22: 548-254, 2004.

22. Friedrich O, Reid MB, Van den Berghe G, Vanhorebeek I, Hermans G, Rich MM and Larsson L: The sick and the weak: Neuropathies/myopathies in the critically ill. Physiol Rev 95: 1025-1109, 2015 